\title{
QUEEN'S
UNIVERSITY
BELFAST
}

\section{Shadowed fading in body-to-body communications channels in an outdoor environment at $2.45 \mathrm{GHz}$}

Cotton, S. L. (2014). Shadowed fading in body-to-body communications channels in an outdoor environment at $2.45 \mathrm{GHz}$. In Proceedings on 2014 IEEE-APS Topical Conference on Antennas and Propagation in Wireless Communications (APWC) (pp. 249-252). Institute of Electrical and Electronics Engineers Inc..

https://doi.org/10.1109/APWC.2014.6905548

Published in:

Proceedings on 2014 IEEE-APS Topical Conference on Antennas and Propagation in Wireless Communications (APWC)

\section{Document Version:}

Peer reviewed version

Queen's University Belfast - Research Portal:

Link to publication record in Queen's University Belfast Research Portal

\section{Publisher rights}

( $) 2015$ IEEE. Personal use of this material is permitted. Permission from IEEE must be obtained for all other uses, in any current or future media, including reprinting/republishing this material for advertising or promotional purposes, creating new collective works, for resale or redistribution to servers or lists, or reuse of any copyrighted component of this work in other works.

\section{General rights}

Copyright for the publications made accessible via the Queen's University Belfast Research Portal is retained by the author(s) and / or other copyright owners and it is a condition of accessing these publications that users recognise and abide by the legal requirements associated with these rights.

Take down policy

The Research Portal is Queen's institutional repository that provides access to Queen's research output. Every effort has been made to ensure that content in the Research Portal does not infringe any person's rights, or applicable UK laws. If you discover content in the Research Portal that you believe breaches copyright or violates any law, please contact openaccess@qub.ac.uk. 


\section{Shadowed Fading in Body-to-Body Communications Channels in an Outdoor Environment at $2.45 \mathrm{GHz}$}

\begin{abstract}
In this paper a number of outdoor body-to-body communications channels at $2.45 \mathrm{GHz}$ which are deemed to be susceptible to shadowed fading are analyzed. The newly proposed shadowed $\kappa-\mu$ model is used to characterize these channels. Its probability density function is shown to provide an improved fit to the distribution of the signal fading compared to established models such as lognormal, Nakagami and Rice.
\end{abstract}

Simon L. Cotton

\section{INTRODUCTION}

Shadowing of the received signal in body centric communications channels is known to be one of the most important factors in determining their fading characteristics. This is particularly the case for bodyto-body (B2B) communications which occur when one or more wireless devices situated on one person communicate with wireless devices situated on other persons in the local vicinity [1-5]. In B2B channels, the direct signal path between the bodies may be obscured by one or even both of users' bodies leading to the phenomenon known as dual node shadowing. This can cause the B2B link to be lost altogether even at very short separation distances of a few meters [6] Moreover, this situation may be further exacerbated when B2B communications operate in populated environments where other persons and obstacles may also intersect the main signal propagation path.

A new statistical fading model for shadowed body centric communications channels was recently proposed in [6]. In this model, clusters of multipath are assumed to have scattered waves with identical powers, alongside the presence of elective dominant signal components - a scenario which is identical to that observed in $\kappa-\mu$ fading [7]. The difference between the model proposed in [6] and that of $\kappa-\mu$ fading is that the resultant dominant component, formed by phasor addition of the individual dominant components is assumed to be a log-normally distributed.

In this paper, the body-to-body channels presented in [6] are characterized using an alternative fading model, namely the shadowed $\kappa-\mu$ fading model, in which the lognormal distribution is superseded by the Nakagami- $m$ distribution. This method of modeling shadowed fading has recently and independently been proposed in [8] and [9]. Unlike [6], it has the attractive feature that its probability density function (PDF) can be expressed in closed-form. The performance of the shadowed $\kappa-\mu$ fading model for modeling the fading characteristics of the received signal in B2B communications channels is then compared with traditional fading models such as lognormal, Nakagami and Rice which are commonly applied in the literature.

\section{EXPERIMENTAL SETUP AND MEASUREMENTS}

\subsection{Experimental setup}

The bodyworn nodes used in this study consisted of the body sensor node (BSN) platform developed by Imperial College London and presented in [6]. The transceiver section of the node utilized a Texas Instruments CC2420, which has a linear dynamic operating range of approximately $100 \mathrm{~dB}$, a maximum transmit power of $0 \mathrm{dBm}$ and a receive sensitivity of $-95 \mathrm{dBm}$. A transmitter node was configured to generate a continuous wave signal with a power level of $0 \mathrm{dBm}$ at $2.45 \mathrm{GHz}$ and a receiver node which was programmed to record the 8-bit received signal strength indicator (RSSI) obtained from the CC2420 every 16 ms. As shown in Figure 1, the BSN nodes were modified to replace the on-board chip antenna with a flexible patch antenna which had a gain of $+6.2 \mathrm{dBi}$ and $\mathrm{a}-10 \mathrm{~dB}$ bandwidth of approximately $55 \mathrm{MHz}(2.398-2.453 \mathrm{GHz})$.

\subsection{Measurements}

The experiments conducted here were performed in an outdoor playing field at the Victoria Park recreational facilities in Belfast, United Kingdom (Figure 1). The measurement environment consisted of a level grass play area which was bounded on three sides by trees and shrubbery and situated beside a soccer pitch adjoining on the remaining side. The area over which the measurements were performed was at least $50 \mathrm{~m}$ from each of these boundaries. The transmit and receive nodes were attached without the use of a dielectric spacer to the central chest region of two adult males of height $1.95 \mathrm{~m}$ and mass $105 \mathrm{~kg}$ (person $\mathrm{A}$ ) and $1.82 \mathrm{~m}$ and mass $95 \mathrm{~kg}$ (person $\mathrm{B}$ ) respectively using a small strip of Velcro ${ }^{\circledR}$. As shown in Figure 1, the units were mounted directly on the test subject's clothing so that the ground plane of the antenna was parallel to the body surface.

A number of different usage scenarios which were considered to be particularly vulnerable to body shadowing were analyzed. The first of these were rotational measurements where persons $\mathrm{A}$ and $\mathrm{B}$ first 

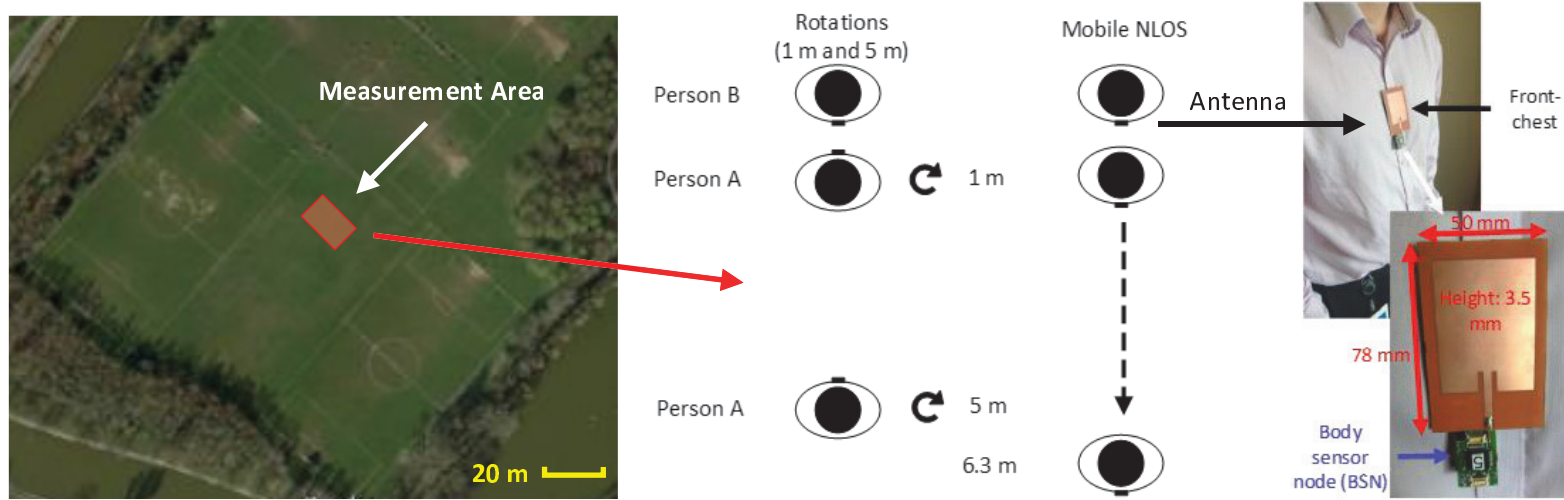

Figure 1: Measurement environment, scenarios and setup.

stood facing one another with a separation distance of $1 \mathrm{~m}$. Person A then performed a full $360^{\circ}$ rotation, moving from direct LOS through to complete nonLOS (NLOS) before returning to an LOS orientation. This procedure was also repeated for a separation distance of $5 \mathrm{~m}$.

The second set of measurements considered the scenario when Person A was mobile, walking directly away from Person B who was stationary. In this instance, Person A started at a point $1 \mathrm{~m}$ from Person $B$ such that the direct signal path between the antennas was obscured by Person A's body (this scenario is herein referred to as mobile NLOS). Due to the received signal regularly extending below the receive sensitivity of the body sensor nodes, only the first $6 \mathrm{~s}$ of person A walking in NLOS from person B were used in the analysis presented here. Given the walking speed of approximately $0.88 \mathrm{~m} / \mathrm{s}$, this was equivalent to Person A walking a distance of 5.3 meters.

\section{SHADOWED FADING MODEL AND DATA ANALYSIS}

A statistical characterization of the body-to-body channels utilized in [6] was performed using the shadowed $\kappa-\mu$ model presented in [8] alongside the lognormal, Nakagami and Rice models. The probability density function of the fading signal in the shadowed $\kappa-\mu$ model is given in equation (1), where $\kappa$ is related to $\delta, \sigma$ and $\mu$ through the relationship $\kappa=\delta^{2} / 2 \mu \sigma^{2}$, which is simply the ratio of the total power of the dominant components $\left(\delta^{2}\right)$ to the total power of the scattered waves $\left(2 \mu \sigma^{2}\right)$ where $\mu$ is related to the multipath clustering and the mean power is given by $\hat{r}^{2}$. In (1), $\Gamma(\bullet)$ is the gamma function. Of course it is worth reinforcing that unlike the $\kappa-\mu$ model of [7], here $\delta$ varies according to Nakagami- $m$ distribution where $m=E^{2}\left[\Delta^{2}\right] / \operatorname{var}\left[\Delta^{2}\right]$ is the Nakagami parameter and $\operatorname{var}\left[\Delta^{2}\right]$ is the variance of the dominant component. In this instance, $\Omega=E\left[\Delta^{2}\right]$ is the average power of the resultant dominant component. For convenience, the rms signal level, $\hat{r}=\sqrt{E\left[R^{2}\right]}$, or in the case of the NLOS mobile measurements, the free space path loss, was removed from the fading envelopes. All parameter estimates for the PDF of the shadowed $\kappa-\mu$ model were then obtained using a nonlinear optimization algorithm written in MATLAB. Parameter estimates for the lognormal, Nakagami and Rice PDFs were obtained using the mle function also available in the Statistics Toolbox of MATLAB.

$$
f_{R}(r)=\frac{2 r^{2 \mu-1}}{\Gamma(\mu)}\left(\frac{\mu(1+\kappa)}{\hat{r}^{2}}\right)^{\mu}\left(\frac{m \hat{r}^{2}}{\mu(1+\kappa) \Omega+m \hat{r}^{2}}\right)^{m} \exp \left(-\frac{\mu(1+\kappa) r^{2}}{\hat{r}^{2}}\right){ }_{1} F_{1}\left(m ; \mu ; \frac{\Omega(\mu(1+\kappa) r)^{2}}{\hat{r}^{2}\left(\mu(1+\kappa) \Omega+m \hat{r}^{2}\right)}\right)
$$




\section{RESULTS}

Figures 2 and 3 show the shadowed $\kappa-\mu$ PDF fitted to the empirical PDFs obtained from the rotational measurements made at the $1 \mathrm{~m}$ and $5 \mathrm{~m}$ points respectively. Also shown for comparison are the best fitting lognormal, Nakagami and Rice PDFs whose parameters were estimated using the procedure described above. As we can quite clearly see, the shadowed $\kappa-\mu$ PDF provides a significantly improved fit to the data obtained from shadowed B2B fading channels compared to the Rice PDF.

Also from Figures 2 and 3, it can be seen that the lognormal and to a much lesser extent the Nakagami PDFs can follow the general shape of the empirical data. For the rotational measurements conducted with a $1 \mathrm{~m}$ separation distance between the two bodies (Figure 2), the fit of the shadowed $\kappa-\mu$ PDF at signal levels below the mean is very good, however its performance at higher signal levels is less satisfactory. Here, the Nakagami PDF provides a better description of the distribution of the data. Nonetheless, it is often the deepest fades that wireless systems designers are most interested in as these can be most detrimental to the quality of the radio link.

Table 1 presents the parameter estimates for the shadowed $\kappa-\mu$ model over all of the rotational measurements. As we can see on both occasions, the $\kappa$ parameters here are very high suggesting that the vast majority of the signal power is carried in the resultant dominant component. The corresponding $m$ parameters are low $(m \rightarrow 0)$ which intuitively suggests that the resultant dominant component undergoes very significant shadow fading. This is expected because as the user rotates, their body intersects the direct signal path causing it to be increasingly attenuated as their body moves from direct LOS to direct NLOS conditions and then back again.

For the mobile NLOS measurements (Figure 4), the shadowed $\kappa-\mu$ distribution again provided a very good fit to the empirical data. Both the Nakagami (at low signal levels) and lognormal (above the mean signal level) distributions also provided a reasonable fit in this scenario. From Table 1 , it can be seen that the $\kappa$ parameter decreases for this scenario and the $\mu$ parameter increases compared to the rotation scenario. This suggests that, as would be expected, the resultant dominant component is significantly reduced, because the user's body continuously obstructs the LOS signal path. From the increase in the $\mu$ parameter it can be inferred that a number multipath clusters contributed to the fading observed in the channel. The estimated $m$ parameter of the shadowed $\kappa-\mu$ model was greater than that obtained for the rotation scenario suggesting that the variation of the resultant dominant component was reduced compared to the rotation scenario.

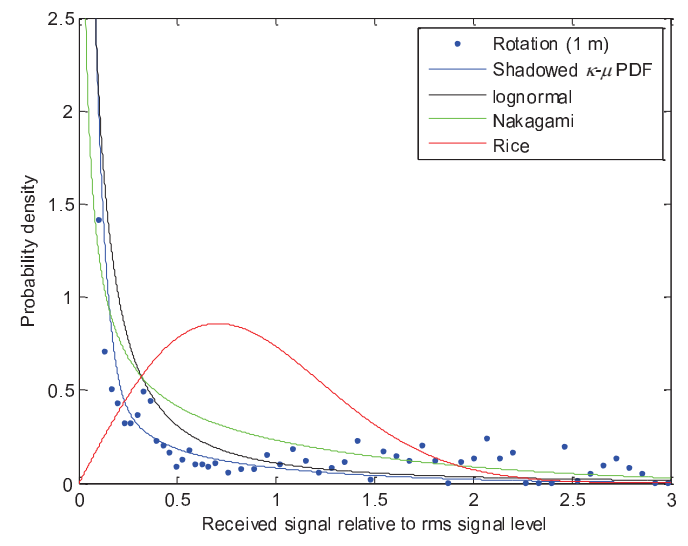

Figure 2: Shadowed $\kappa-\mu$, lognormal, Nakagami and Rice PDFs fitted to the empirical PDF for B2B channels while person A rotated at a distance $1 \mathrm{~m}$ from person B.

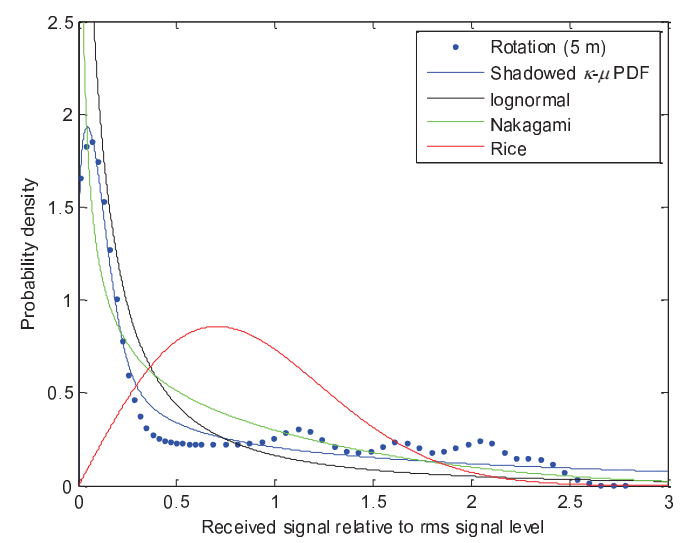

Figure 3: Shadowed $\kappa-\mu$, lognormal, Nakagami and Rice PDFs fitted to the empirical PDF for B2B channels while person A rotated at a distance $5 \mathrm{~m}$ from person B.

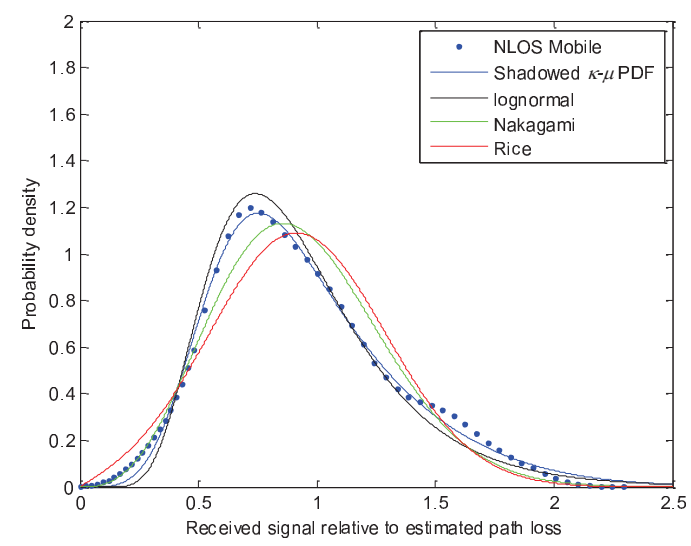

Figure 4: Shadowed $\kappa-\mu$, lognormal, Nakagami and Rice PDFs fitted to the empirical PDF for B2B channels while person A walked away from person B. 


\begin{tabular}{|c|c|c|c|c|}
\hline Scenario & Shadowed $\kappa-\mu$ & lognormal & Nakagami & Rice \\
\hline \multirow{2}{*}{ Rotation $(1 \mathrm{~m})$} & $\kappa=431.5, \mu=0.25, \hat{r}=1.24$, & $\alpha=-2.18$, & $v=0.17$, & $A=0.03$, \\
& $m=0.04, \Omega=0.37$ & $\beta=1.89$ & $\omega=1.00$ & $s=0.71$ \\
\hline \multirow{2}{*}{ Rotation $(5 \mathrm{~m})$} & $\kappa=141.9, \mu=0.56, \hat{r}=1.31$, & $\alpha=-1.45$, & $v=0.25$, & $A=0.03$, \\
& $m=0.18, \Omega=5.00$ & $\beta=1.64$ & $\omega=1.00$ & $s=0.71$ \\
\hline \multirow{2}{*}{ Walking (NLOS) } & $\kappa=1.73, \mu=2.81, \hat{r}=1.10$, & $\alpha=-0.14$, & $v=1.90$, & $A=0.84$, \\
& $m=0.62, \Omega=0.63$ & $\beta=0.39$ & $\omega=1.00$ & $s=0.39$ \\
\hline
\end{tabular}

Table 1: Parameters estimates for each of the fading models fitted to the measurement data. For the lognormal $\mathrm{PDF}, \alpha$ is the mean and $\beta$ is the standard deviation of the fading signal's logarithm, for the Nakagami PDF, $v$ is the shape parameter and $\omega$ is the mean power, for the Rice PDF, $A$ is the non-centrality parameter and $s$ is the scale parameter.

\section{CONCLUSION}

In many situations, the direct signal path in body-tobody communications channels will be subject to shadowing caused by the user's bodies and obstacles in the local surroundings. Therefore channel models which can accommodate fading due to shadowing as well as multipath propagation need to be considered. To this end, the shadowed $\kappa-\mu$ fading model has been used to characterize a number of body-to-body communications channels operating at $2.45 \mathrm{GHz}$. It has been shown that this new shadowed fading model can provide an improved fit to empirical data compared to existing fading models such as lognormal, Nakagami and Rice.

\section{Acknowledgments}

This work was supported by the U.K. Royal Academy of Engineering and the Engineering and Physical Research Council (EPSRC) under Grant Reference EP/H044191/1 and by the Leverhulme Trust, UK through a Philip Leverhulme Prize.

\section{References}

[1] S. L. Cotton and W. G. Scanlon, "Channel characterization for single and multiple antenna wearable systems used for indoor body-to-body communications," IEEE Trans. Antennas \& Propagation, Special Issue on Antennas \& Propagation in Body-Centric Wireless Communications, vol. 57, no. 4, pp. 980-990, April 2009.

[2] Y. Wang, I. B. Bonev, J. O. Nielsen, I. Z. Kovacs and G. F. Pedersen, "Characterization of the indoor multiantenna body-to-body radio channel," IEEE Transactions on Antennas and Propagation, vol. 57, no. 4, pp. 972-979, April 2009.
[3] R. Rosini, R. D'Errico and R. Verdone, "Bodyto-body communications: A measurement-based channel model at $2.45 \mathrm{GHz}, "$ IEEE 23rd International Symposium on Personal Indoor and Mobile Radio Communications (PIMRC), pp.1763-1768, 9-12 Sept. 2012.

[4] S. L. Cotton, A McKernan and W. G. Scanlon, "Received signal characteristics of outdoor bodyto-body communications channels at $2.45 \mathrm{GHz}$," Loughborough Antennas and Propagation Conference (LAPC), pp. 1-4, November 2011.

[5] S. L. Cotton, W. G. Scanlon, E. Skafidas and B. K. Madahar, "Millimeter-wave stealth radio for special operations forces," Microwave Journal, Military Microwaves Supplement, vol. 53, no. 8, pp. 6-16, Aug. 2010.

[6] S. L. Cotton, "A statistical model for shadowed body centric communications channels: theory and validation," IEEE Transactions on Antennas and Propagation, vol. 62, no. 3, pp. 1416-1424, March 2014.

[7] M. D. Yacoub, "The $\kappa-\mu$ and the $\eta-\mu$ distribution," IEEE Antennas Propagation Magazine, vol. 49, no. 1, pp. 68-81, Feb. 2007.

[8] S. L. Cotton, "Human Body Shadowing in cellular device-to-device communications: channel modeling using the shadowed $\kappa-\mu$ fading model," under revision, IEEE Journal on Selected Areas in Comms Special Issue on Device-to-Device Comms in Cellular Networks, 2013.

[9] J. F. Paris, "Statistical characterization of $\kappa-\mu$ shadowed fading," IEEE Transactions on Vehicular Technology, vol. 63, no. 2, pp. 518526, February 2014. 